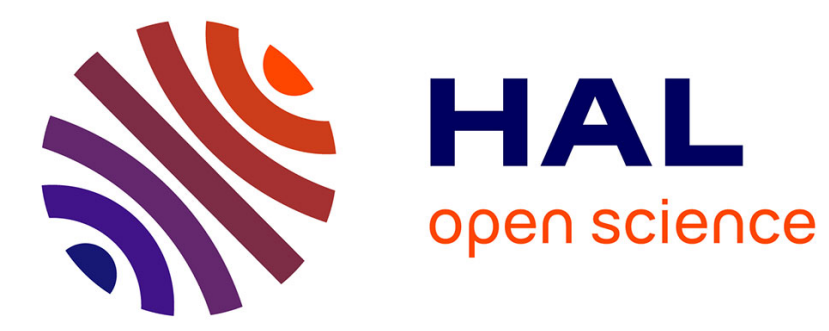

\title{
THE LOCAL SPIN-DENSITY-FUNCTIONAL METHOD : APPLICATION TO THE METAL-INSULATOR TRANSITION IN DOPED SEMICONDUCTORS
}

\author{
A. Ghazali, P. Leroux-Hugon
}

\section{To cite this version:}

A. Ghazali, P. Leroux-Hugon. THE LOCAL SPIN-DENSITY-FUNCTIONAL METHOD: APPLICATION TO THE METAL-INSULATOR TRANSITION IN DOPED SEMICONDUCTORS. Journal de Physique Colloques, 1980, 41 (C5), pp.C5-317-C5-323. 10.1051/jphyscol:1980552 . jpa-00219987

\section{HAL Id: jpa-00219987 https://hal.science/jpa-00219987}

Submitted on 1 Jan 1980

HAL is a multi-disciplinary open access archive for the deposit and dissemination of scientific research documents, whether they are published or not. The documents may come from teaching and research institutions in France or abroad, or from public or private research centers.
L'archive ouverte pluridisciplinaire HAL, est destinée au dépôt et à la diffusion de documents scientifiques de niveau recherche, publiés ou non, émanant des établissements d'enseignement et de recherche français ou étrangers, des laboratoires publics ou privés. 
THE LOCAL SPIN-DENSITY-FUNCTIONAL METHOD : APPLICATION TO THE METAL-INSULATOR TRANSITION IN DOPED SEMICONDUCTORS,

A. Ghazali and P. Leroux_Hugon

Laboratoire de Physique des Solides CNRS, 92190 Meudon, France.

Résumé.- On expose les principes de la méthode de la fonctionnelle de la densité locale de spin ainsi que son utilité pour étudier les systèmes d'électrons en interaction, en particulier ceux mettant en jeu des moments magnétiques localisés; différentes applications de la méthode sont indiquées. On passe en revue les arguments expérimentaux en faveur de l'existence de moments localisés sur les impuretés chargées dans les semiconducteurs dopés. Utilisant le formalisme de la fonctionnelle de la'densité, on décrit la transition isolant-métal et la disparition de la polarisation locale de spin qui ont lieu lorsque la concentration en impuretés augmente.

\begin{abstract}
The principles of the Local Spin-Density-Functional (LSDF) method, its usefulness to study interacting electron systems, especially those involving local magnetic moments, and its various applications are outlined. Experimental evidences for magnetic moments localized on charged impurities in doped semiconductors are briefly reviewed. The insulator-to-metal transition and the vanishing of local spin polarization, which occur as the impurity concentration increases, are described using the LSDF formalism.
\end{abstract}

1. Introduction.- In the field of magnetism or in the one of semiconductors, a number of problems amounts to find for each specific class of electronic systems (or of materials) a convenient way to deal with electron-electron interactions, i.e. with manybody problems. To tackle these problems, a strategy is to transform them into a onebody problem, that of an electron submitted to an effective potential suitably defined and self-consistent. The effective mass approximation $/ 1 /$ and the approach by schrieffer $/ 2 /$ and cyrot $/ 3 /$ of the Hubbard problem arc examples of such a strategy. This type of method has proved itself to be of particular value for problems like the one of metal-insulator transition and of change of magnetic properties which take place in systems such as some transition metal oxides. The aim of this paper is to present a method in which exchange and correlation contributions, to the electron-electron interactions are accounted for through an effective potential, this (one-body) effective potential depends only on the local charge and spin densities and is defined in a selfconsistent way, This method, termed the Local Spin Density Functional (LSDF), has a wide range of applicability. Its advantage, in eg. the problem of metal-insulator transition and of local magnetic moment formation, is that, when compared to the Hubbard method, it introduces neither an oversimplified scheme nor an adjustable parameter.

Section 2 and 3 of this paper present the principles of the method and some of its applications. In section 4 , we briefly review the experimental data for the specific problem of the metal-insulator transition in doped semiconductors and of its magnetic aspects, and we discuss how to apply the LSDF method to this problem in section 5, Some prospects of application of the method to other magnetic semiconductor problems are outlined in section 6 .

2. Principles of the LSDF method.- The density functional method relies on a theorem by Hohenberg and $\mathrm{Kohn}^{/ 4 /}$. These authors have shown that (i) the energy of an interacting electron system is a unique functional of its local density $n(\vec{r})$ and that (ii) this energy is minimal for the actual density of the system. Within this functional, one singles out a contribution which is specifically due to exchange and correlation interactions, $E_{x c}[n(\vec{r})]$. This has led Kohn and sham $/ 5 /$ to show that, for a given system, the problem of determining the electron states reduces to the solution of a set of Schrödinger-like wave equations : $\left\{-\frac{\hbar^{2}}{2 m} \nabla^{2}+v_{0}(\vec{r})+e^{2} \int \frac{n\left(\vec{r}^{\prime}\right)}{\left.\mid \vec{r}-\vec{x}^{2}\right) \mid} d \vec{r}^{2}+v_{x c}[n(\vec{r})]\right\} \psi_{i}(\vec{r})=$ $\varepsilon_{i} \psi_{i}(\vec{x})$ 
for a particle in presence of an effective potential which incorporates an exchangecorrelation term $v_{x c}$

$$
v_{x c}[n(\vec{r})]=\frac{\delta^{E} x[n(\vec{r})]}{\delta n(\vec{r})}
$$

The density $n(\vec{r})$ itself is given $b_{y}$ a selfconsistency relation

$$
n(\vec{r})=\sum_{i}|\psi(\vec{r})|^{2}
$$

the sum running over the occupied states of the system. In (1), enter the kinetic ener$g y$, the external potential $v_{0}(\vec{r})$ and the Hartree potential. By solving the wave equation, one gets the internal energy of the system. The method is generalized to the case of finite temperature, giving access to the free energy and thus to the thermodynamics of the system.

In general, the form of $E_{x c}[n(\vec{r})]$ is not known. Interest of the method lies in the possibility, for slowly varying $n(\vec{r})$, to approximate $\mathrm{E}_{\mathrm{Xc}}$ by the integral of a local quantity.

$$
E_{x c}[n(\vec{r})]=\int n(\vec{r}) \varepsilon_{x c}[n(\vec{r})] d \vec{r}
$$

where $\varepsilon_{\mathrm{xc}}$ is the exchange-correlation energy per electron of a uniforme electron gas with density $n$. Then

$$
v_{x c}[n]=\frac{d}{d n}\left(n \varepsilon_{x c}[n]\right)
$$

and the internal energy $\mathrm{E}$ is

$$
\begin{gathered}
E=\sum_{i} \varepsilon_{i}-\int \frac{1}{2} \frac{n(\vec{r}) n\left(\overrightarrow{r^{\prime}}\right)}{\left|\left(\vec{r}-\vec{r}^{\prime}\right)\right|} d \vec{r} d \vec{r}^{\prime}+\int n(\vec{r}) \\
\left\{\varepsilon_{x c}[n(\vec{r})]-v_{x C}[n(\vec{r})]\right\} d \vec{r}
\end{gathered}
$$

The density functional method has been generalized to account for the spin polarisation 16,7/: within the local approximation, one introduces, beside the local charge density $n(\vec{r})$, the local spin density or local spin polarization $p(\vec{r})$. The exchange correlation energy $\varepsilon_{x c}$ and the associated potential $v_{x c}$ are then functional of $n(\vec{r})$ and $p(\vec{r})$, both quantities being defined by self-consistency relations. This extension of the method is particularly useful to describe the formation of local moments $/ 7 /$.
The preceding relations are the constitutive equations of the ISDF method in which a key role is played by the local exchange-correlation energy $\varepsilon_{x c}[n(\vec{r}), p(\vec{r})]$. Within this scheme, the study of a given electron system, specified by the external potential $v_{0}(\vec{r})$, reduces to the self-consistent determination of $\mathrm{n}(\overrightarrow{\mathrm{r}})$ and $\mathrm{p}(\overrightarrow{\mathrm{r}})$. Let us point out that this method, devised to calculate the internal energy, does not lend itself, a priori, to the determination of the excitation energies in every situation.

3. Application range of the LSDF method.Application of the LSDF method have been stimulated by the availability of a parametrized form for $\varepsilon_{x c}[n(\vec{r}), p(\vec{r})] / 7 /$. This parametrization, derived from the most careful studies of the uniform electron gas, are precise in a rather wide range of electron density. The use of the method for atomic calculations, $/ 7 /$ has shown it to be valid beyond the bounds set by the use of the local approximation (slowly varying electron density). Being applied to solids, $/ 7,8 /$ the method has yielded valuable results as regarlas e.g. interatomic distances or cohesive energies. In the case of magnetic systems, the LSDF has been useful to compute spin-polarized band structures $/ 9,10 /$ it has allowed some authors $/ 11 /$ to discuss the problem of orbital and spin configuration and of their evolution with interatomic distances $/ 11,12 /$ Gunnarsson and Lundqvist $/ 7 /$ have pointed out the interest of the method to discuss spontaneous symmetry breakings, for instance the breaking of the spin symmetry in the $\mathrm{H}_{2}$ molecule as the internuclei distance exceeds a critical value. This strongly suggests to apply this method to problems of metal-insulator transition and of associated magnetic configuration changes.

Another aspect of the method is its usefulness to study linear responses of non-uniform systems : dielectric constant $/ 4,13 /$ and magnetic susceptibility $/ 5 /$. It has been shown $/ 7 /$ that the LSDF formalism is relevant to estimate the non-diagonal susceptibilities $x^{(m, e)}=x^{(e, m)}$, i.e. the spin density fluctuation response to a charge density fluctuation and vice-versa; these 
quantities are known to play an important role in the study of the magnetic polaron $114 /$. Vosko and perdew / $15 /$ have thoroughly discussed the spin susceptibility of a nonuniform electron system and their method have been successfully applied to estimate the Stoner enhancement of the Pauli susceptibility of metals (including transition metals) and to discuss the occurrence of ferromagnetic instability /16/.

There have been attempts to improve the description of electronic systems in which the density is not slowly varying anywhere, by avoiding those errors which are due to the use of the local approximation. Rather than gradient expansions, non-local methods based upon the consideration of the exchange-correlation hole $/ 17,18 /$ seem promising. Let us notice that errors due to the local approximation are in general more important on excitation energies than on the internal energy of the system (see the extreme case of the hydrogen atom discussed in ref/19/.

Finally, beside its use in ab initio calculations, the density functional method may be combined with the pseudopotential approximation $/ 20 /$ or with the effective mass approximation $/ 21 /$. The application of the LSDF to thie case of doped semiconductors which we will discuss in section 5 , has been worked out within the effective mass approximation.

Let us emphasize again that one of the major advantages of the LSDF is the fact that it lends itself to thermodynamical calculations.

4. Metal-insulator transition and local moment formation: the case of doped semiconductors.- An example of metal-insulator transition in which electron correlation are likely to be important and in which the change of electronic properties is accompanied by local magnetic moment formation is the one of doped semiconductors, especially hydrogenic systems for which the effective mass approximation is relevant. An advantage of these systems is that the parameter responsible for the transition, the charged impurity concentration, may be varied continuously over a wide range, but a drawback is the effect of disorder, associated with the non-periodic distribution of impurities, which may blur the electron correlation effects. Let us notice that these problems of extrinsic transitions are raised in usual semiconductors (e.g. P-doped $s i$ ) as well as in magnetic semiconductors (e.g. Euo with oxygen vacancies).

The metal-insulator transition manifests itself by a variation of the low temperature resistivity by many orders of magnitude over a narrow range of concentration /22/. The insulating (or semiconducting) state is specified by the occurrence of an activation energy of the conductivity. A first activation energy, $\varepsilon_{1}$, corresponds to the excitation of an electron from the localized ground state, termed $D^{\circ}$, to the conduction band; $\varepsilon_{1}$ decreases as the donor concentration increases. Under some conditions (doping range, temperature), one identifies a second activation energy $\varepsilon_{2}$, which also depends on donor concentration $/ 23 /$. This activation energy is commonly attributed to the excitation of an electron from the $D^{\circ}$ ground state to a localized excited state, termed $\mathrm{D}^{-}$, made of two electrons with opposite spins located on the same impurity site. As the concentration increases, a broadening of impurity bands takes place and the states $\mathrm{D}^{\circ}$ and $\mathrm{D}^{-}$give rise to the lower and to the upper Hubbard band respectively/24/. Within this hypothesis, the discussion of $\varepsilon_{2}$ regime and the question to ascertain whether the transition comes about from the vanishing of $\varepsilon_{1}$ or from the one of $\varepsilon_{2}$, requires an appropriate description of the spin configuration of $D^{\circ}$ and $D^{-}$.

Far in the insulating regime, an electron localized on a charged impurity center has a well defined spin $1 / 2$ magnetic moment; far in the metallic regime, the electron gas is spin unpolarized. In other words, the orbital delocalization is accompanied by a spin delocalization although the two transitions do not take place at the very same concentration $/ 25 /$. This change of spin configuration has been evidenced by the following phenomena :

(i) Evolution of the ESR spectrum. In e.g. P-doped silicon, there is a change from a spectrum with two narrow lines, sepa- 
rated from each other by the hyperfine coupling with the impurity nucleus, which is typical of localized states, to a spectrum with a single narrow line, typical of a metallic system $/ 26 /$.

(ii) Evolution of the spin susceptibility $X$, deduced from d.c. measurements or from integration of the ESR line. It changes from a curie or Curie-Weiss behaviour, owing to the antiferromagnetic exchange coupling with the neighbours, with $x$ proportional to the donor concentration, to a Pauli one, possibly exchange enhanced, with $X$ proportional to $\mathrm{N}_{\mathrm{D}} 1 / 3 / 28 /$.

(iii) Evolution of the NMR signal (Knight shift) $/ 29 /$.

(iv) Evolution of the Faraday rotation and spin-flip-Raman-scattering spectra /30/.

In order to interpret some of these experiments, some authors $/ 28,30 /$ have suggested the coexistence, in a narrow concentration range, of localized and extended states. Recent experiments emphasize the importance in the neighbourhood of the transition, of exchange coupling between randomly distributed moments $/ 31,32 /$ (spin-glass behaviour),

These magnetic aspects of the transition point to the need of a method which accounts not only for the evolution of the local. electron density (i.e. the localized. to-extended state transition) but also for the spin density one; this is precisely what the I.SDF method is able to do.

\section{Application of the LSDF method to the} metal-insulator transition in doped semiconductors. - To discuss this transition, an ambitious programme would be to compute, from first principles, the evolution of the electronic structure in an impurity system embedded in the semiconductor matrix, However, as far as the effective mass approximation is valid, the problem amounts to the much simpler discussion of an hydrogen atom system, with a proper scaling (effective mass and dielectric constant leading to an affective Rydberg and an effective Bohr radius). We shall address ourselves to this problem and especially to the description of interactions between electrons originating from donors, within the LSDF formalism. Beside electron-electron interactions, an important aspect of the problem is the overlap of wave functions centered on adjacent impurity sites. As the impurity concentration increases, this overlap leads to the formation of an impurity band. We shall deal with this aspect of the problem, by assuming the existence of a pseudo-regular lattice of impurities, within the wigner-seitz approximation. In so doing, we neglect the effects associated with the non-periodic distribution of impurities (e.g. Anderson transition). Let us notice also that, as the IDSF method lends itself more easily to describe local moment formation than to account for their correlations, we will not discuss the problem of magnetic ordering.

In ref./33/, we have applied the LSDF method to the problem of doped semiconductors, stressing the spin polarization change and the closing of the gap between localized and extended states, as the impurity concentration increases. We have solved the KohnSham equation by a variational method. To be specific, we have introduced trial wave fuctions :

$$
\left.\begin{array}{l}
\psi_{n, k}(\vec{r})=u_{n, k}(\vec{r}) \text { e }(i \vec{k}, \vec{r}) \\
u_{n, k}(\vec{r})=A\left[v_{n, k}^{(0)}(\vec{r})-i \vec{k} \cdot \vec{r} \cdot v_{n, k}^{(I)}(\vec{r})\right] \\
\text { with : } v_{n, k}^{(0)}(r)=\exp (-\alpha r)+B\left(1-\frac{\alpha r}{2}\right) \exp (-\alpha r / 2) \\
v_{n, k}^{(1)}(r)=\exp (-\alpha r)+B\left(1+\frac{\alpha a_{0} r_{s}}{4}-\frac{\alpha r}{2}\right) \exp (-\alpha r / 2)
\end{array}\right\}
$$

where $A$ is the normalization constant. Combination of $1 \mathrm{~s}-\mathrm{like}$ and $2 \mathrm{~s}$ - like functions allows us to impose the Wigner-Seitz conditions which define $B$. The $v(1)$ function accounts for the formation of bands via the $\vec{k} \cdot \vec{p}$ method. The variational parameter, $\alpha \equiv$ $a_{n, k}$, is defined through the minimisation of the energy $\varepsilon_{n, k}$ associated to $\psi_{n, k}$ ' the system of $\psi_{n, k}$ satisfying the self-consistency relations. The set of wave equations depends on the impurity concentration $N_{D}$ throuhg the radius of the wigner-seitz sphere $a_{0} r_{s}\left(a_{0}\right.$ is the effective Bohr radius),

$$
\frac{4}{3} \pi a_{0}^{3} r_{S}^{3} N_{D}=1
$$


$A$ and $B$ on one hand, $\alpha$ on the other hand depend on $r_{s}$. This trial function lends itself to describe a localized state ( $\left.\alpha a_{0}{ }\right)$ ) and an extended one $\left(\alpha a_{0}<1\right)$. The variational calculation clearly displays these two types of solutions. This is illustrated in figure 1 which shows the variational energy $\varepsilon$ as a function of the reduced parameter $z=\alpha a_{0}$ for a value of $r_{s}$ corresponding to a metallic state in our model $\left(r_{\mathrm{s}}\right.$ $=3.14$ or $\mathrm{n}=0.470$, where the reduced concentration $n=N_{D} / N_{0}$ and $N_{0}=\pi / 3\left(4 a_{0}\right)^{3}$. The difference between the two kinds of solutions is further evidenced in figure 2 which shows the radial distribution of electron density within the Wignre-seitz sphere, for two impurity concentrations corresponding to systems with respectively fully localized $(n=0.212)$ and fully extended $(n=0.470)$ solutions. Finally, the dispersion curves of these two kinds of solutions are strongly different and lead to quite different densities of states (See Fig 3 below).

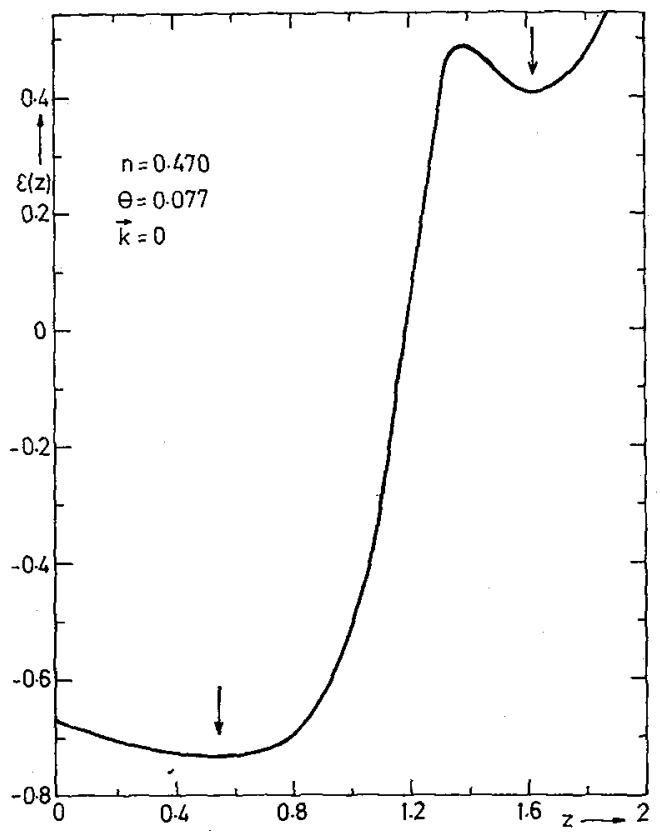

Fig. 1 Variational energy $\varepsilon$ as a function of the reduced parameter $\mathrm{z}$ at $\vec{k}=0$, for the reduced concentration $n=0.470$. The energy is given in effective Rudbexgs (Ry) and the concentration in units of $\mathrm{N}_{0}=\pi / 3\left(4 \mathrm{a}_{0}\right)^{3}$. Axrows show the position of the minima corresponding to the extended (small z) state and to the localized (large $z$ ) state; $\theta=k_{B} T / R Y$.

The variational calculation, selfconsistently performed, shows that, at low concentration, the stablest state is the localized, fully spin polarized one, whereas at high concentration, the extended state for which the effect of spin polarization is small, is the stablest. Localized solutions build up a narrow impurity band and extended solutions build up the perturbed conduction band.

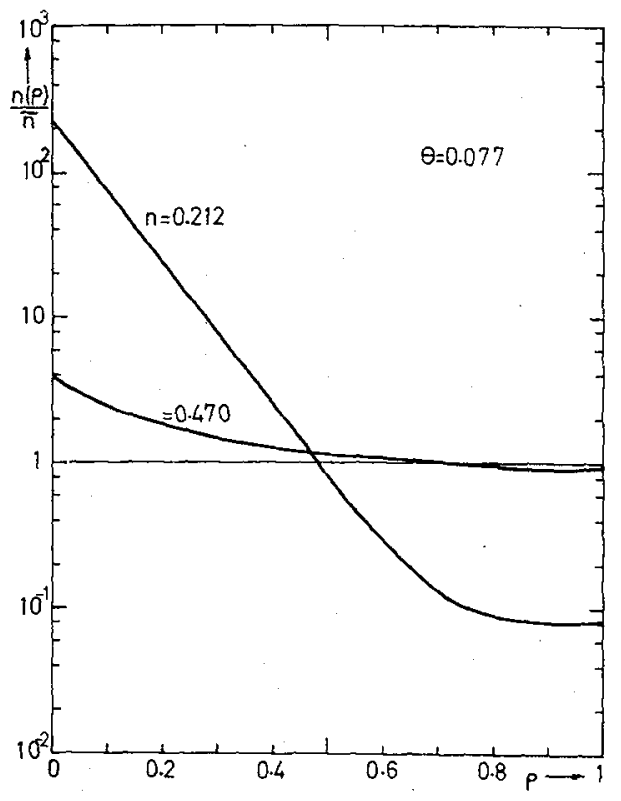

Fig. 2 Electron density $\mathrm{n}(0)$ in units of the average density $\vec{n}$ plotted in logarithmic scale as a function of the reduced radius $\rho=\frac{x}{a_{0} r_{s}}$ for two concentrations, $n=0.212$ and $n=0.470 ; \theta=k, T / R y$. Note the fulfiliment of the Wigner-Seitz conditions.

The insulator-to-metal transition corresponas to the crossing of these bands. This calculation predicts, in a narrow concentration and temperature range for which the Fermi level crosses both bands, the coexistence of (narrow) impurity band states and (wide) conduction band states much like in the Friedel-Anderson scheme. Figure 3 displays, for concentrations corresponding to typical insulating, semi-metallic and metallic regimes, the densities of states and the filling of bands, computed following this model.

The ISDF method, as we have implemented it, does account for the two major features of the transition, the orbital delocalization and the spin delocalization (vanishing local spin polarisation). This result emphasizes the importance of electron-electron 
interaction mechanisms. Our treatment leads also to a thermodynamical analrsis of the transition.

However our solution, as any variational solution, depends to some extent on the trial functions we have used.

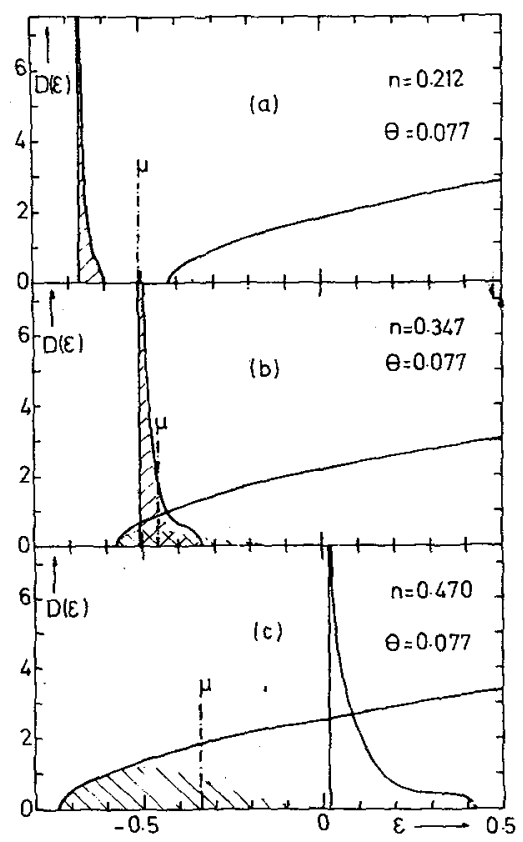

Fig. 3 Evolution of the density of states $D(\varepsilon)$ the impurity and conduction bands as the donor concentration $n$ increases for (a) an insulating, (b) a semimetallic and (c) a metallic configuration (from ref. 33). $\mu$ is the Fermi level and $\theta$ is the temperature. Hatched areas correspond to occupied states. The concentration is in units of $N_{0}=\pi / 3\left(4 a_{0}\right) 3, D(\varepsilon)$ in uni ts of $N_{0} / R y$ and energy in RY ; $\theta=k_{B} T / R y$.

In particular, one may show that the variation of the localized state energy with concentration we have found is too rapid, owing to the way we have introduced the WignerSeitz conditions. Thus one may hope to improve the solution both quantitatively (e.g. the value of the critical concentration for the transition) and qualitatively by making the trial function more flexible as regards the orbital and spin degrees of freedom.

One may also solve the set of wave equations by othex methods as Rose et al. /34/ have done. These authors have shown a transition from a fully polarized ground state to a partialiy polarized one to occur at $r_{s}=2.84$ and a second transition from this partially polarized ground state to an unpolarized one at $r_{s} \doteq 2.74$. Improvements of the solution would allow a more detailed discussion of problems such as the spin structure of the $D^{-}$state and the concentration dependence of $\varepsilon_{2}$. Finally, we remark that the problem of disorder and its consequences on transport properties (Anderson transition) and magnetic ones (amorphous antiferromagnetism) is still open.

6. Conclusion.- We have discussed an application of the LSDF method to a specific problem of magnetic semiconductor physics; we would like now to suggest some other applications in this field, applications which, we believe, would be worth studying.

A first application is the computation of spin polarized band structures in materials like EuO or $\mathrm{CaCr}_{2} \mathrm{~S}_{4}$, to compare them to existing experimental and theoretical data. A second application is the study of (intrinsic) metal-insulator transition in transition metal oxides (highly correlated systems) like $\mathrm{VO}_{2}$ and $\mathrm{V}_{2} \mathrm{O}_{3}$ : advantages of the method here lie on its ability to provide a thermodynamical discussion and to take into account lattice energies. Very recently, skriver et al. $135 /$ have reported on such a study performed on transition-metal monoxide series from Cao to NiO) accounting for the occurrence of antiferromagnetically ordered moments and of insulating behavior (i.E. Mott insulators). In the third place, starting from a suitable modelisation, the LSDF method is likely to provide a thorough analysis of the magnetic polaron formation (stability, shape of the charge and spin densities distributions) in the case of e.g. oxygen vacancies doped Euo. Finally, as the method lends itself to discuss orbital and spin configurations and their evolution with interatomic distances, it would be most useful to study mixed valent materials to which this configuration changes is the central problem. 


\section{References}

1/ Kohn, W., Phys. Rev. 105 (1957) 508 Ibid. 110 (1958) 857 .

12/ Evenson, W., Schrieffer, J., and Wang, S., J. Appl. Phys. 41 (1970) 1199.

/3/ Cyrot.M., J. Physique (Paris) 33 (1972) 125.

/4/ Hohenberg, P., and Kohn, W., Phys. Rev. 136, B (1964) 864 .

/5/ Kohn, W., and Sham, L., Phys. Rev. 140 A (1965) 1133 .

/6/Von Barth, U., and Hedin, L., J. Phys. C 5 (1972) 1629 .

/7/ Gunnarsson, $0 .$, and Lundqvist, B. , Phys. Rev. B 13 (1976) 4274 .

/8/ Tong, B., Phys. Rev. B 6 (1972) 1189.

19/ Callaway, J., and Wang, C., Phys. Rev. B 16 (1977) 2095 .

/10/ Gunnarsson, O., J. Appl. Phys. 49 (1978) 1399.

/11/ Skriver, H., Andersen, O., and Johansson, B., Phys. Rev. Lett. 4 I (1978) 42.

/12/ Glötzel, D., J. Phys. F 8 L (1978) 163 .

/13/ Hedin, L., and Lundqvist, B., J. Phys. C $\underline{4}$ (1971) 2064 .

/14/ Leroux-Hugon, P., Phys. Rev. Lett. 29 (1972) 939.

115/ Vosko, S., and Perdew, J., Can. J. Phys. 53, (1975) 1385 .

/16/ Janak, J., Phys. Rev. B 16 (1977) 255.

117/ Gunnarsson, O., Jonson, M., and Lundqvist, B., solid state Commun. 24 (1977) 765 .

/18/ Alonso, J., and Girifalco, L., Phys. Rev. B 17 (1978) 3735 .

/19/ Gunnarsson, O., Lundqvist, B. ; and wilkins, J., Phys. Rev. B 10 (1974) 1319.

/20/ Moriarty, J., Phys. Rev. B. 16 (1977) 2537.

/21/ Rose, J., Shore, H., and Zaremba, E., Phys. Rev. Lett. 37 (1976) 354.

/22/ Yamanouchi, C., Mizuguchi, K., and Sasaki, W., J. Phys. Soc. Japan 22 (1967) 859.

/23/ Review in Fritzsche,H., The Metal NonMetal Transition in Disordered Systems, Friedman, . $_{\text {, }}$ and TunstalI, D., edit (Scottish Universities Summer School in physics, 1978) p. 193.

/24/ Review in Kamimura, H., ref /23/p. 327 .

/25/ Review in Holcomb, D., ref /23/ p. 251 .

/26/ Morigaki, K., and Maekawa, s. J. Phys. Soc. Japan 32 (1972) 462 .

/27/ Sasaki, W., and Kinoshita,J., J. Phys. Soc. Japan 25 (1968) 1622 .
128/ Quirt, J., and Marko, J., Phys. Rev. B 5 (1972) 1716 and 7 (1973) 3842 .

/29/ Sasaki, W., Ikehata, S., and Kobayashi, S., J. Phys. Soc. Japan 36 (1974) 1377 and Ikehata, S., Sasaki,W., and Kobayaski, S., J. Phys. Soc. Japan 39 (1975) 1492 .

/30/ Geschwind, S., Romestain, R., Hu, D., Devlin, G., and Jedju, T., Semicond. Insul. 4 (i978) 1 .

/31/ Walstedt, R., Kummer,R., Geschwind, s., Narayanamurti,V., and Devlin, G., J. Appl. Phys. 50 (1979) 1700 .

/32/ Andres, K., Bull. Amer. Phys. Soc. 24 (1979) 262 .

133/ Ghazali, A., and Leroux-Hugon, P., Phys. Rev. Lett. 41 (1978) 1569.

/34/ Rose, J., Sander, L., Shore, H., and Chakravarty, S., Bull. Amer. Phys. Soc. 24 (1979) 280 and to be published. We thank these authors for making their results available to us prior to publication.

/35/ Skriver, H., Andersen, O., and Johansson, B., paper 5 B 2, intern. Conf. Magnetism, Munich 1979 (to appear in J. Magn. Magn. Mat.) 